\title{
RETRACTED ARTICLE: Personal distress, but not sympathy, predicts the negative influence of bystanders on responding to an emergency
}

\author{
Ruud Hortensius • Dennis J. L. G. Schutter • Beatrice de Gelder
}

Published online: 3 December 2014

(C) Psychonomic Society, Inc. 2015

At the request of the authors this article has been retracted. During the preparation of a follow-up study, a mistake was found in the experimental script of the cued reaction time task of experiment 2. Four out of six conditions were mislabeled. Consequently, the reported findings and their interpretation and discussion are incorrect. Careful reexamination and reanalyzing of the data using the correct labels revealed a pattern of results that is not entirely compatible with several of the main claims of the article. Importantly, the corrected results show that reaction times do not increase with more bystanders present at an emergency. Moreover, not only personal distress but also perspective taking predicts the negative influence of bystanders on reaction times. We believe that these new findings undermine our central claim of decreased action preparation as a function of bystanders present at an emergency and the enhancement of this effect in people with higher levels of trait personal distress. While the results and discussion of experiment 1 and 3 remain correct, the new results of experiment 2 influence the article to such an extent that we currently see no other option than to retract the article from publication. We will continue to work on this topic and hope to publish the new results in due time.

We deeply regret the publication of invalid results. We sincerely apologize to the Editor and reviewers of the manuscript, and the readers of Cognitive, Affective, \& Behavioral Neuroscience.

R. Hortensius $\cdot$ B. de Gelder $(\bowtie)$

Cognitive and Affective Neuroscience Laboratory,

Tilburg University, Tilburg, The Netherlands

e-mail: b.degelder@maastrichtuniversity.nl

\section{J. L. G. Schutter}

Donders Institute of Brain, Cognition and Behaviour,

Radboud University Nijmegen, Nijmegen, The Netherlands

B. de Gelder

Brain and Emotion Laboratory, Department of Cognitive

Neuroscience, Faculty of Psychology and Neuroscience, Maastricht

University, Maastricht, The Netherlands

B. de Gelder

Brain and Emotion Laboratory Leuven, Division of Psychiatry,

Department of Neurosciences, KU Leuven, Leuven, Belgium 